\title{
Algunas categorías y preguntas para el aula de literatura'
}

\author{
Some categories and questions for the literature classroom
}

\author{
ANALÍA GERBAUDO \\ Consejo Nacional de Investigaciones \\ Científicas y Técnicas-Universidad Nacional del Litoral \\ Argentina \\ analiagerbaudo7ı@yahoo.com.ar
}

Resumen. Este artículo reúne categorías y preguntas que en diferentes niveles (teórico, metodológico y epistemológico) le permiten al profesor que enseña literatura en el nivel medio interrogar sus decisiones, reflexionar sobre ellas y convertirlas en objeto de experimentación didáctica.

Las categorías son "aulas de literatura", "obstáculo epistemológico", "obstáculo ideológico", "buenas prácticas" y "docente como autor del curriculum". La primera describe las elecciones didácticas en un aula de literatura; para ello se formulan preguntas que, lejos de prescribir, ayudan a analizarlas. Las tres siguientes juegan por contraste: oponer los obstáculos de orden ideológico y epistemológico a las buenas prácticas desarma el círculo normativo al desconstruir la idea de lo "lo bueno" como parámetro haciendo lugar a un concepto que contenga las tensiones involucradas en la enseñanza de un objeto artístico como la literatura. Finalmente, la última repone el lugar del profesor en el diseño del aula.

Palabras clave: aulas de literatura; obstáculo epistemológico; obstáculo ideológico; buenas prácticas.
Abstract. This article brings together categories and questions which allow secondary school literature teachers to review their classroom decisions at different levels -theoretical, methodological and epistemic-, and reflect upon them and turn them into the object of educational experimentation.

The categories selected for this article are "literature classroom," "epistemic interference", "ideological hindrance", "good teaching practice" and "the teacher as the author of the curriculum." The first one aims to describe the didactic decisions taken in the literature classroom; to do that, some questions are asked which, far from being prescriptive, help to analyze daily options. The next three offer a contrast: by opposing ideological and epistemic obstacles to good practice, the normative circle is dismantled, because it is possible to deconstruct the idea of what is "good" as a parameter, bringing in instead a concept that contains the tensions involved in the teaching of an artistic object such as literature. Finally, the last one reinstates the place of the teachers within the classroom lay-out.

Keywords: literature classroom; epistemic obstacle; ideological obstacle; good teaching practice.

\footnotetext{
${ }^{\text {I }}$ Para citar este artículo: Gerbaudo, Analía (20I3). Algunas categorías y preguntas para el aula de literatura. Alabe 7. [www. revistaalabe.com]

(Recibido 24-O5-20I2; aceptado o8-OI-2OI3)
} 


\section{Tres investigaciones y algunos resultados}

Los resultados que se condensan en este artículo se derivan de tres proyectos de investigación desarrollados en y sobre Argentina. El primero, centrado en el análisis de las articulaciones y los desajustes entre la Teoría Literaria, las propuestas curriculares oficiales y los manuales destinados a la enseñanza de la literatura para el nivel medio durante el contexto de la reforma educativa de los años noventa² ${ }^{2}$ Más allá de la descripción de los materiales y de su análisis, el trabajo dio lugar a la elaboración de un conjunto de preguntas de orden epistemológico que permiten discutir qué es posible o deseable que acontezca en un aula de literatura: sin desatender los lineamientos ministeriales juega allí un lugar central el docente como "autor del curriculum" (cf. Gerbaudo, 20o6). Los dos proyectos siguientes estuvieron centrados en el mapeo del estado actual de la enseñanza de la lengua y de la literatura en la escuela secundaria ${ }^{3}$. De ese cartografiado (cf. Gerbaudo, 2OII) me detengo especialmente en lo referido a la enseñanza de la literatura y, puntualmente, en los aportes categoriales producidos dado que aportan a una didáctica que se imagina reflexiva y no prescriptiva, teórico-epistemológica y no recetual, en diálogo con la perspectiva que desde México, tras el análisis de las secuelas de las reformas implementadas en ese país desde 1997 en adelante, delinea Díaz Barriga (20o9). Básicamente se cuestiona el desplazamiento de los planteos didácticos que, tanto en Argentina como en México, privilegiaron una visión de "corte tecnocrático-eficientista" preocupada por la "calidad" y la "eficiencia” educativas (medidas según los parámetros de las evaluaciones nacionales e internacionales) unida al desprecio de todo "teoricismo": "se pretende que quien aspira al título de profesor aprenda en la práctica, bajo la supervisión de un maestro en ejercicio, los conceptos que subyacen en estas disciplinas", afirma Díaz Barriga. Y agrega: “‘Abajo el teoricismo!’, significó en la reforma educativa ‘¡Arriba el empirismo y el practicismo"” (2009: 24). Esta propuesta parte de la revisión crítica de esos supuestos.

\section{Las categorías y las preguntas (o las bases de una didáctica de la literatura no prescriptiva)}

“Obstáculo epistemológico”, la clásica categoría de Gaston Bachelard (i948) retomada desde el campo de la didáctica por Alicia Camilloni (1997) para estudiar transferencias de conceptos científicos, contribuyó a definir las búsquedas de investigación

\footnotetext{
${ }^{2}$ Tesis de Maestría en Didácticas Específicas (UNL): Análisis teórico-epistemológico de las articulaciones y desarticulaciones entre la teoría literaria y las propuestas curriculares y editoriales para la Educación General Básica 3. Implicancias para la enseñanza de la literatura. Publicada en 2006 (cf. Bibliografía).

3 Obstáculos epistemológicos en la enseñanza de la lengua y de la literatura en el nivel EGB3: notas para una agenda didáctica actualizada. CÅI+D 2005-2008 (FHUC-UNL) y Obstáculos epistemológicos y buenas prácticas en la enseñanza de la lengua y de la literatura en la escuela secundaria: diagnóstico y propuestas de intervención. CAI+D 2009-2OI2 (ambos bajo mi dirección).
} 
a partir del segundo proyecto. Si el obstáculo epistemológico, bajo la forma de sabercristalizado, genera inercias que dificultan la reflexión sobre el objeto, es necesario detectar cuáles son los que afectan al campo de la enseñanza de la literatura para tratar de descolocarlos teniendo presente que jugará en contra de este procedimiento la tendencia a confirmar lo que se cree más que la alerta respecto de lo que contradice dichas creencias. Cuando en el marco de una comunidad no aparecen interrogantes sino sólo respuestas, o cuando una se fija (a modo de ritual o receta), "el obstáculo epistemológico se incrusta sobre el conocimiento que ya no se cuestiona" (Camilloni i3). Por ejemplo, una joven profesora que ejerce en escuelas del nivel medio dice en una entrevista: "No me parece interesante enseñar literatura desde el estructuralismo; pasa que si no lo hago me da como culpa". En el comentario se entreveran cuestiones de varios órdenes: el saber de la profesora como lectora choca con su formación profesional a la vez que revela una zona inexplorada y un mandato que naturaliza un punto de vista que no es más que uno entre otros. Lo cristalizado imposibilita elegir: el desafío es desplazar la sensación de que se está "traicionando" un legado para traer las razones que fundamentan por qué se lo soslaya. Es la densidad de la literatura y sus derivas posibles en la configuración de otras subjetividades lo que está en juego.

Muchos de los obstáculos epistemológicos registrados en las aulas de literatura del nivel secundario tenían una base ideológica. Acuñamos entonces el término "obstáculo ideológico" a partir del enlace de los conceptos "obstáculo epistemológico" (Bachelard, I948) e "ideología" (Eagleton, I995). Esta categoría da cuenta de las representaciones que impiden leer debido a cuestiones de orden ideológico que promueven interpretaciones a priori. Así como la ideología es lo que persuade a hombres y a mujeres a confundirse, de vez en cuando, con dioses o con bichos (Eagleton: I5), los obstáculos ideológicos son los que llevan a actuar como "dios" o como "bicho" y/o a colocar a ciertos objetos (textos literarios) como representantes del bien o del mal absolutos.

Ambos obstáculos, ideológicos y epistemológicos, son la principal traba para la reflexión ya que apartan cualquier noción u objeto que desestabilice las rutinas seguidas en las "aulas de literatura", es decir, en esas construcciones que comprenden no sólo el diseño didáctico de las clases sino el conjunto de decisiones previas que se ponen en juego en cada una de ellas a lo largo de un período escolar: la selección de contenidos, de materiales, los corpus (en plural dado que se trabajará con diferentes grupos de textos atados por distintas hipótesis según los contenidos a enseñar durante el ciclo), los "envíos" (es decir, las interpelaciones que conducen a textos que no se incluirán en la “enseñanza oficial” [en el sentido de que no figurarán en el programa] ni en la evaluación pero que se incorporan en las relaciones que establece el docente en sus planteos orales), el diseño de evaluaciones, la "configuración didáctica” (Litwin, I996) de las clases. Este concepto llama la atención sobre el carácter artesanal y complejo de cada propuesta didáctica dado que cada grupo particular de alumnos requiere una re-selección de los contenidos pautados por los Ministerios (nacionales y jurisdiccionales), un ajuste tanto de los textos adecuados a sus posibilidades de lectura como de las categorías y actividades 
empleadas para promoverla. Para cada grupo se diseñarán singulares articulaciones con los materiales complementarios (películas, fotografía, pinturas, música) y cada armado de clases supondrá desafíos imprevistos en función de los avances en los aprendizajes, de los contenidos que es necesario volver a enseñar porque las evaluaciones muestran que no se han aprendido, de los temas que irrumpen desde fuera de la agenda y que pueden incorporarse junto a los planificados. Se pretende generar una relación vital entre escuela y resto de la vida: un espacio y un tiempo sensibles a "aquellos elementos vivos que se hallan en el entorno social, cultural y escolar del estudiante” (Díaz Barriga, 20o9: 29).

Este concepto de "aula de literatura" se anuda con el de "buena práctica". Entre Edith Litwin, Gary Fenstermacher y Gilles Deleuze, este constructo lee las intervenciones de los docentes haciendo foco en su cuerpo, en el entramado subjetivo que los lleva a tomar las decisiones teóricas y epistemológicas que atraviesan sus diseños. Insisto en el "entre" ya que si bien fue Fenstermacher quien definió el concepto de "buena enseñanza", las investigaciones de Litwin lo introdujeron en Argentina: sus estudios sobre las configuraciones didácticas del aula universitaria (I997) o su definición de los ejes de la "nueva agenda didáctica" (I996) retoman la tesis de Fenstermacher que liga la "buena enseñanza” con la justificación teórica y epistemológica del docente respecto de la importancia de que sus alumnos "conozcan, crean o entiendan" un determinado contenido. Se advertirá que la carga ideológica de cada verbo varía y actualiza, cada vez, distintas representaciones de la práctica en un arco que va de la transferencia de conocimientos a la militancia pasando por la comprensión.

El concepto de "buena práctica" enlaza a Fenstermacher con Deleuze para hacer lugar al análisis de cada decisión a partir del cuerpo de quien la toma (el cuerpo es el parámetro o la medida). Esta inclusión desenmascara el carácter subjetivo del objeto de enseñanza. Dice Deleuze: "Lo bueno tiene lugar cuando un cuerpo compone directamente su relación con el nuestro y aumenta nuestra potencia con parte de la suya. Lo malo tiene lugar cuando actúa como un veneno que descompone la sangre" (I981: 33). Incluir esta variable en el concepto de "buena práctica" supone reconocer el territorio que ocupan la indeterminación, la experiencia y la subjetividad (Kuri, 2007: 362) en las escenas educativas: el docente realiza cada vez un armado artesanal que pone a disposición de los estudiantes a los que destina su enseñanza, sin poder garantizar el efecto ni controlar más que la acreditación (acreditar y aprender, vale recordarlo, son procesos diferentes). Sí puede analizar cada una de sus decisiones al tallar los objetos: no hay punto neutro ni conmensurabilidad que habilite a medir si es mejor enseñar literatura desde la narratología (Roussin, 20IO), la sociología (Sapiro, I999, 20II) o la ecocrítica (Glotfelty y Fromm, I996; Coupe, 2000; Garrard, 2004), entre otras4. Hay en cada caso un docente-lector que elige atendiendo a razones no sólo teóricas y epistemológicas sino también políticas, estéticas (el viejo problema del "gusto” es imposible de esquivar tratándose de literatura)

4 Elijo citar trabajos actuales enmarcados en estas teorías. Para una reconstrucción de los antecedentes históricos y del estado del arte de estas y otras líneas, ver Gerbaudo, 2006; Kaufmann, 2OII. 
e ideológicas en las que operan su historia y su subjetividad. Siguiendo con el ejemplo: la narratología, la sociología y la ecocrítica tienen diferentes representaciones respecto de la relación entre la literatura y la vida, la literatura y los sujetos, la literatura y el medio social. Por lo tanto los contenidos a enseñar, las actividades a realizar e incluso los corpus (algunos serán más adecuados para trabajar ciertos contenidos) variarán según el docente se incline por una u otra.

Se sigue de lo anterior que una exigencia de arranque para pensar en "aulas de literatura” asociadas a "buenas prácticas” es que el docente cuente con una formación teórica, literaria y artística suficiente que le permita decidir cómo diseñarlas ya que esto es lo que lo habilitará a “firmar”. En Argentina, un efecto de la reforma de los años noventa fue el borramiento de la marca de autor en cada programa docente unido al hecho de que, por diversas causas imposibles de resumir aquí, en muchas ocasiones las aulas de literatura se han diseñado desde las editoriales de venta de manuales que parametraban desde la selección de contenidos y textos hasta el diseño de las evaluaciones con sus respuestas (cf. Gerbaudo, 2006). Por otro lado, advertir que las didácticas se elaboran a partir de prácticas de los docentes en ejercicio ayuda, mediante analogías y contrastes, a ejercer un control epistemológico sobre los quehaceres y a fomentar la confrontación y el debate respecto de las maneras de encarar el trabajo sin buscar fórmulas ni recetas válidas para todo contexto y caso. Finalmente, mostrar las diferencias de posición sobre la enseñanza de la literatura pone a la vista su carácter controversial y estimula la discusión. No es el fin de este artículo convocar al consenso. Muy por el contrario: busca interpelar a revisar las muy diferentes formas de diseñar didácticamente el objeto de transferencia.

Un objeto que exige atender a cuatro órdenes teóricos básicos cada vez que se lo analiza desde una lente que se quiera didáctica: uno que involucra concepciones acerca del aprender (¿cómo aprenden los estudiantes?), otro que recupera posiciones acerca del enseñar (¿qué cuestiones entran en juego cuando se habla de "buena práctica”?), un tercer orden que atañe a los “credos teóricos” respecto del objeto disciplinar (¿cómo se define a la "literatura"?) y un cuarto que comprende el contexto (la legislación existente, los documentos curriculares y paracurriculares, el proyecto educativo de cada institución, las características socioculturales de la coyuntura, las tensiones de cada corte del presente en el que se ejerce la acción de enseñar).

Atentos a estos órdenes y contra la presencia de dos expresiones frecuentes entre los profesores y maestros ("bajar a las aulas"; "aplicar” tal o cual teoría o método), armamos los conceptos de "apropiación” y de "reinvención categorial situada” por contraste con la normalización de la literatura bajo las formas del "aplicacionismo", del "deteccionismo" y de la "sinécdoque teórica".

El aplicacionismo es la tendencia a ubicar en un método la solución de los problemas de la lectura. Una ilusión que cree garantizado el abordaje de cualquier texto descansando en el empleo lo más fiel posible de cierto número de categorías sin inscribir como variables las que impone el contexto de re-uso (varían los lectores, las circunstancias de lectura, los textos escogidos, etc.). 
El diseccionismo o deteccionismo es la práctica que promueve la mera identificación de recursos en un texto sin recuperar ese trabajo para describirlo o formular hipótesis de lectura. Esto suele ir acompañado de una abstracción de las circunstancias de producción, circulación y/o recepción de la obra.

Finalmente, la sinécdoque lingüisticista acontece toda vez que se exacerba la atención a los procedimientos de un texto literario con independencia del contexto de producción y de recepción. Operación que inevitablemente, y tal como sucede cada vez con cualquier lectura, toma sólo una parte del objeto. Pero en esta ocasión, pretendiendo dar cuenta del todo.

Puede que en una situación de enseñanza aparezcan juntas estas tres operaciones.

Observemos algunos ejemplos de disección, aplicacionismo y sinécdoque lingüisticista tomados de manuales para el nivel secundario producidos a propósito de la reforma de los años noventa en Argentina. Los ejercicios que transcribimos no tienen otro propósito que "pasar" el contenido más que enseñarlo para intervenir la lectura. Así, bajo el capítulo titulado "El romance", se presentan las consignas que transcribo sobre "Cabalga Diego Laínez”: “* Señalen si, en el romance, hay repeticiones léxicas, repeticiones variadas o aliteraciones. / * Expliquen en qué consisten las construcciones paralelas que se encuentran en el texto". La identificación de recursos no va más allá del reconocimiento y no vuelve sobre el texto, es decir, no se utiliza el contenido supuestamente "enseñado" para complejizar, enriquecer o problematizar la lectura sino que se pasa de ese ejercicio a otro de hechura semejante.

En el mismo manual, en el capítulo "La novela", se presenta el "argumento" de Una canción de navidad de Charles Dickens, se transcribe el "Prefacio y una parte importante del segundo capítulo" y se anexan las siguientes consignas: “* Subrayen en el fragmento las oraciones en las cuales el narrador se dirige en segunda persona a los lectores. / ${ }^{*}$ Determinen si el narrador de Una canción de Navidad es equisciente, omnisciente o deficiente. Justifiquen su respuesta con una cita textual”. Tal como en el ejemplo anterior, la detección no se lleva luego hacia conjeturas sobre el texto, sobre los efectos de lectura que tal o cual decisión del escritor produce, etc.

En tensión con estos conceptos se inscribe el de “apropiación”. Pensar la enseñanza desde este lugar exige trabajar para hacer lugar a que el otro arme una respuesta o una nueva pregunta, no "dictada" por quien la provoca. Como bien remarca Jacques Derrida, en las escenas de transferencia, "la cuestión... debe ser la pregunta que se le deja al otro: la respuesta es del otro.” (2OOI: 46 ).

Cabe revisar qué espacio se le deja al otro en un aula de literatura cuando se lo somete a la mecánica repetición de la misma rutina de ejercicios sin otro fin que su resolución. Ejercicios que no integran la información extractada en la lectura del texto completo. Cabe revisar qué se le deja al otro si, a partir de la conjunción entre teoría y literatura, se inventa otra cosa, se abre otro juego, otro derrotero que, sin evitar el trabajo analítico (se haga desde la teoría que se sea), lo incorpora en otro orden permitiendo complejizar la lectura. Por ejemplo, detectar las metáforas en Los dinosaurios (I983) de Charly García 
es un trabajo que, sin lugar a dudas, se verá enriquecido si se vuelve sobre el texto para interpretarlo atendiendo a las circunstancias de producción: el velamiento y el trabajo obtuso sobre el contenido eran estrategias que permitían sortear la censura durante la última dictadura en Argentina.

En esta misma línea, es decir, tratando de plantear preguntas que permitan que sea el destinatario quien arme las respuestas, se anexa una serie que pretende hacer reflexionar al docente sobre los fundamentos teóricos y epistemológicos de cada una de las decisiones didácticas, tomadas y/o por tomar, en el diseño de cada “aula de literatura". A los fines de precisar el análisis, el desagregado lleva a detenerse en acciones concretas:

- ¿Cómo diseña el programa de la materia?: ¿desde qué criterios “fragmenta” el programa en esas unidades y por qué (¿por qué esas unidades son tales, es decir, por qué son condensadoras de sentidos y, en cada caso, de cuáles?) ¿Para cuánto tiempo planifica cada unidad (¿todas tienen asignado el mismo tiempo? ¿por qué?) ¿Cuántas clases integran de modo estimativo cada unidad? ¿Qué balance arroja la relación entre el tiempo asignado y el tratamiento de los contenidos (¿podrá desarrollar los contenidos con el tiempo que estos requieren?)

- ¿Dónde está la estructura potente de su propuesta pedagógica?: ¿en los contenidos en relación con la bibliografía y los materiales seleccionados? ¿En los contenidos en relación con las clases? ¿En los contenidos en relación con las actividades? Finalmente: ¿considera adecuado plantear estas preguntas en términos disyuntivos o las preguntas solicitan la conjunción "y” en su formulación? ¿Por qué?

- ¿Cómo piensa el diseño de las clases?: ¿todas las clases de su materia tienen el mismo estilo? ¿Plantea clases de apertura o introductorias, de presentación de información, de cierre, de recuperación del error al devolver evaluaciones, etc.? ¿Deben planificarse diferentes estilos de clases según los contenidos a enseñar? ¿Por qué? ¿Distingue las "clases" de las "tutorías"?

- ¿Cómo piensa lo que "hace" en las clases de "literatura"?: ¿qué enseña en las clases de "literatura”? ¿Enseña "literatura"? ¿Es posible enseñar "literatura" o enseña a leer de una manera en función de su posición teórica? ¿Enseña a leer de una manera o enseña a "aplicar" categorías de la teoría literaria? ¿Enseña a leer literatura o usa la literatura para ilustrar contenidos de otras materias? ¿Qué otras cosas enseña mientras enseña "literatura"?

- ¿A qué apuntan sus actividades?: ¿fijan información? ¿Se orientan a la resolución de problemas? ¿Proponen ejercicios de aplicación? ¿Alguna de sus actividades propone una reflexión de tipo epistemológica? ¿Alguna apunta a un trabajo metacognitivo? ¿Por qué?

- ¿Cómo trabaja con los materiales educativos?: ¿selecciona manuales? ¿Antologías? ¿Prepara sus materiales en función de las características de sus estudiantes? ¿Por qué? ¿Pone la literatura en diálogo con otras formas del arte (cine, pintura, música, fotografía, escultura, etc.)? ¿Por qué? 
- ¿Cómo piensa las preguntas que formula?: ¿abren o clausuran posiciones sobre un problema? ¿Plantean relaciones con otros saberes, con otras disciplinas, con problemáticas socioculturales? ¿Chequean la información adquirida por el alumno en relación a una cuestión puntual?

- ¿Cómo piensa la evaluación?: ¿como un trabajo que le posibilita al alumno pensar su propio proceso de aprendizaje ya que le permite identificar qué sabe y qué no sabe, qué aprendió y que le falta aún aprender de lo ya enseñado? ¿Como un apéndice de la enseñanza, necesaria para colocar una calificación en base a lo acreditado por el estudiante? ¿Como parte del proceso de enseñanza y de aprendizaje, en perspectiva de continuidad, es decir, tomando en consideración lo aprendido y las dificultades evidenciadas por los alumnos para re-evaluar lo que se seguirá enseñando (entre lo que se incluye lo que tal vez sea necesario volver a enseñar)? En sus aulas de literatura, ¿transparenta los criterios de corrección que seguirá en la evaluación? Pasado el examen, ¿vuelve sobre los errores, sobre las dificultades?

\section{- ¿Para quéenseña literatura?}

Deliberadamente, el último ítem resta abierto. Sin guías, sin orientaciones. Si en los puntos anteriores puede entreverse la opción elegida por quien escribe las preguntas, en este último caso se deja que, recursivamente, quien lee vuelva sobre las anteriores, enredándolas como en un "bucle extraño" (Hofstadter, I979). Por esa misma razón se ubica hacia el final, deseando que lleve a repasar las respuestas dadas y también las categorías que aquí se propusieron con la fantasía de impedir cualquier fijación de una respuesta como "la" respuesta didáctica para toda aula de literatura.

\section{La investigación como mediación}

El sentido de difundir las categorías y las preguntas aquí planteadas es instalarlas, no sólo entre los profesores sino también en los espacios de formación de formadores. Si bien se sabe que lo que se enseña no es equiparable a lo que se aprende y que los tiempos de la enseñanza no necesariamente coinciden con los del aprendizaje, abrir el diálogo sobre estos puntos así como ampliar los cartografiados de obstáculos epistemológicos, ideológicos y buenas prácticas de diferentes territorios, zonas o regiones ayudaría a instalar una discusión didáctica actualizada y situada sobre la enseñanza de la literatura. Una discusión sustentada en los razonamientos, en la posición argumentada que permite interpretar cada una de las decisiones de aula, tomadas o por venir, pero siempre localizadas en tiempo y espacio.

Sin pretender construir una suerte de "nueva metodología", se intenta también dejar una perspectiva a partir de la cual debatir las representaciones y los fundamentos teóricos y epistemológicos de lo que se enseña en las aulas de literatura ya que sobre esta base se construye el resto de las decisiones didácticas sobre las que aquí se llama la aten- 
ción. Se intenta, en definitiva, instalar una serie de categorías que tocan puntos neurálgicos del tejido didáctico ya que pretenden desatar sus nudos prescriptivos y normativos a partir de la interrogación teórica y epistemológica. Acción que solicita respuestas desde un lugar tensionado entre lo íntimo y la responsabilidad pública asumida junto al ejercicio de nuestro trabajo. Ese que nos ocupa cada uno de todos nuestros días.

Se sabe que lo que pueden los investigadores y sus espacios de publicación comparado con lo que pueden los medios masivos no es mucho, pero es algo. Y bajo esas condiciones escribimos: entre poner las cosas en cuestión y el silencio confirmatorio del estado de las cosas, elegimos lo primero. Allí reside la clave de nuestra mediación y de este envío.

Y hablo de la investigación como mediación así como hablo de la docencia como mediación: si sólo soy un maestro ante los ojos de quien cree que puedo enseñarle cosas que no sabe (Jackson, 1998), del mismo modo los papeles de investigación, para incidir, requieren confianza por parte de quienes leen. La confianza de que tenemos algo para decir y de que lo que tenemos para decir puede derivar en un re-direccionamiento positivo, individual o colectivo. Sin la pretensión de revolucionar las prácticas de enseñanza de la literatura pero tampoco con la falsa modestia o el cinismo de quien pone a circular bajo su firma artículos que cree que no provocarán ningún cambio, apostamos a una transformación gestada desde la jugada plena al tipo de trabajo didáctico a realizar desde cada aula de cada escuela. 


\section{Referencias bibliográficas}

- Bachelard, Gaston (1948): La formación del espíritu científico. México: S. XXI, I980.

- Camilloni, Alicia (1997): Los obstáculos epistemológicos en la enseñanza. Barcelona: Gedisa.

- Coupe, Laurence (2000): The green studies reader:from Romanticism to ecocriticism. London: Routledge.

- Deleuze, Gilles (r98I): Spinoza:filosofía práctica. Barcelona: Tusquets, 200I.

- Derrida, Jacques (200I): “A corazón abierto”. jPalabra! Instantáneas filosóficas. Trotta: Madrid. $13-48$.

- Díaz Barriga, Ángel (2009): Pensar la didáctica. Buenos Aires: Amorrortu.

- Eagleton, Terry (1995): Ideología. Una introducción. Barcelona: Paidós, I997.

- Fenstermacher, Gary (I986): “Tres aspectos de la filosofía de la investigación sobre la enseñanza”. La investigación de la enseñanza, I. Enfoques, teorías y métodos. Merlin Wittrock, editor. Barcelona: Paidós. I48-I59.

- Garrard, Greg (2004): Ecocriticism. New York: Routledge.

- Gerbaudo, Analía (2006): Ni dioses ni bichos. Profesores de literatura, curriculum y mercado. Santa Fe: Universidad Nacional del Litoral.

- --. (20п) La lengua y la literatura en la escuela secundaria. Santa Fe-Rosario: Universidad Nacional del Litoral-Homo Sapiens.

- Glotfelty, Cheryll y Harold FROMM (I996): The ecocriticism reader: landmarks in literaryecology. Georgia: University of Georgia Press.

- Hofstadter, Douglas (1979): Gödel, Escher, Bach un Eterno y Grácil Bucle. Barcelona: Tusquets, 1998.

- Jackson, Philip (1998): "Sobre el lugar de la narrativa en la enseñanza”. La narrativa en la enseñanza, el aprendizaje y la investigación. Hunter Mac Ewan y Kieran Egan, compiladores. Buenos Aires: Amorrortu. 25-5I.

- Kaufmann, Vincent (2OII): La faute à Mallarmé. L'aventure de le théorie. Paris: Du Seuil.

Kuri, Carlos (20O7): Estética de lo pulsional. Lazo y exclusión entre psicoanálisis y arte. Santa Fe: UNL. 
- Litwin, Edith (1996): "El campo de la didáctica: la búsqueda de una nueva agenda”. Corrientes didácticas contemporáneas. Alicia Camilloni, compiladora. Buenos Aires: Aique. 9I-II5.

- --. (1997): Las configuraciones didácticas. Una nueva agenda para la enseñanza superior. Buenos Aires: Paidós.

- Roussin, Philippe (2OIO) : « Généalogies de la narratologie, dualisme des théories du récit ». Narratologies contemporaines. Nouvelles approches pour la théorie et l'analyse du récit. Paris : Éditions des Archives contemporaines. 45-73.

- Sapiro, Gisèle (1999) : La guerre des écrivains (1940-1953). Paris : Fayard.

---. (2OII) La responsabilité de l'écrivain. Littérature, droit et morale en France (XIX-XX siècle). Paris: Du Seuil.

- Zizek, Slavoj (1997): Elacoso de lasfantasías. México: S. XXI, I999. 